\title{
LXIV. Investigation of certain remarkable and unexplained phænomena of vision, in which they are traced to functional actions of the brain
}

\section{Mr. Thomas Smith}

To cite this article: Mr. Thomas Smith (1832) LXIV. Investigation of certain remarkable and unexplained phænomena of vision, in which they are traced to functional actions of the brain , Philosophical Magazine Series 3, 1:5, 343-349, DOI: 10.1080/14786443208647912

To link to this article: http://dx.doi.org/10.1080/14786443208647912

曲 Published online: 01 Jun 2009.

Submit your article to this journal $\pi$

Џll Article views: 1

Q View related articles $\sqsubset$ 
bring the bubble of the additional level by means of its pinion exactly to between the two marks drawn across its tube*. Continue thus successively to depress the telescope by the pinion $L$ until its bubble stands at the reversing point, and afterwards to elevate it by the pinion $\mathbf{J}$ until the bubble of the additional level comes to between its marks; and when the repetition has been carried far enough, the altitude may be found by dividing the mean of the two readings by the number of observations.

Apparently, there would be no difficulty in obtaining any multiple of the double zenith distance of a celestial object procured precisely as by the French circle. Troo additional levels, both fixed to the divided circle, would then be indispensable; one to be levelled when the instrument had been turned half round in azimuth, and the other when the telescope had been pointed the second time at the star.

By means of the two additional levels, the difference of zenith distance of two objects might be measured at once, without obtaining the absolute zenith distance of either.

Leeds, Sept. 4, 1832.

J. Nixon.

Erratum.-Page 108, line 1, for $11^{\prime \prime}$ read 11'.

LXIV. Investigation of certain remarkable and unexplained Phanomena of Vision, in rohich they are traced to Functional Actions of the Brain. By Mr. Thomas Smith, Surgeon, Fochabers.

[Concluded from p. 258.]

$\mathbf{H}$ AVING thus ascertained the precise nature of the effects, the next step I took was to investigate the true nature of the exciting cause. In all the experiments in which I had hitherto observed the phænomena, the light which appeared to excite them was more or less rohite; for the bright object was either a lamp or candle, or the direct rays of the sun, or the sun's light reflected from snow or the like. In addition to this circumstance, the bright object had always been so situated in relation to the point $\mathrm{P}$, to which the eyes were accommodated, that its image on the retina must have been formed either

* It would be preferable to have both levels fitted up with accurate scales, and to note the position of their bubbles rather than to attempt to bring them always to one tixed mark. From the register of the deviations of the bubble of the telescope from its reversing point, and those of the bubble of the additional level from the point at which it stood when the telescope pointed at the star, it can be ascertained how many seconds are to be added to or subtracted from the final reading. 


\section{Mr. T. Smith's Investigation of certain Phanomena of}

before or behind the true focal point. Now, as in the investigation of new phænomena, with the principles of which we are, as in the present case, utterly unacquainted, every combination of the circumstances ought, as far as possible, to be tried till we arrive at the most simple which is capable of producing the results; so it appeared proper to try if any one kind of light, or any one position of it, was more efficacious than another in exciting the appearances. The following experiment, repeated often with the utmost care, convinced me that an imperfect image of the bright object was required to fall on the retina in order to produce the phænomena.

Exp. 8. I placed a strong bright light at the nearest distance to which my vision could adapt itself, and directing both eyes to it, I caused a screen to be interposed between one of them and the light; so that one of my eyes only was exposed to the bright light, and the image of it was formed perfectly distinct on the retina. A slip of rohite paper, illuminated from behind me, was held so near my eyes as to appear double; the result was very remarkable. Of the two images, that which was seen by the exposed eye appeared darker than that which was seen by the shaded eye; but both appeared distinctly white, without any tinge whatever of green or red. In performing this experiment, great caution is required that the exposed eye be adapted correctly to the distinct vision of the flame; for by much observation I have found that a small error in this respect, such as occurs when the eye becomes dazzled, is sufficient to excite those changes in the sensibility to red light, which have been proved to be the causes of the green and red appearances of the white paper.

The difference of brightness observed in the two images in this experiment is undoubtedly owing to the operation of the affection of sight, mentioned in Note *, p. 255, \&c. as may be proved by shading or exposing both eyes, by turns. When the images appear unequally bright, by shading both eyes, the darker image acquires the same luminousness as the brighter one; and by exposing both, the bright image becomes of the same shade as the dark one.

Having thus ascertained that bright light failed in eliciting the phænomena when it formed a distinct image on the retina, it remained to try the effects of different kinds of light. The results of numerous observations carefully made with the different primary colours, are shown in the following experiment.

Exp. 9. I raised a broad yellow flame in the manner recommended by Sir David Brewster for the construction of a monochromatic lamp: this I placed in the position F, fig. 1, near my right eye, and applied a tube, blackened within, to my 
other eye, to prevent the result from being disturbed by any stronger light entering my left eye. A lighted candle was placed in a dark lantern behind me, with only a small opening in it to permit a stream of white light to fall on the slip of white paper $\mathrm{S}$, which was placed so as to be visible to both eyes when they were directed to a distant point $P$. The experiments, with the other primary colours, were made with narrow tubes of thin coloured paper. One of the tubes being applied to the right eye, was strongly illuminated by means of lights placed near its sides; and a black tube was applied to the left eye, to insure thai inequality of the action of the coloured light on the two eyes, which, even in a more moderate degree, had been found sufficient with rohite light to produce the phænomena: the results in all these trials were striking and uniform. The image of $S$, seen by the eye exposed to the primary coloured light, was constantly of the colour that was complementary to that of the tube or light employed,-an appearance manifestly referrible to the affection of sight mentioned in Note *, p. 255, \&c.; but the image of $\mathrm{S}$ that was seen through the black tube was uniformly rohite, being never in the smallest perceptible degree changed by the affection of the other eye.

From these observations we learn that no excess of any primary coloured light entering one of the eyes is able to produce the affection which we have been investigating; hence it follows that white light only is capable of exciting it. But the 8th experiment proves that white light also fails to produce it, when it forms a distinct image on the retina. It is not the action of the white light, therefore, but the indistinctness of the white image, that constitutes the true exciting cause.

This conclusion leads us to remark, that the affection of vision now under investigation, as well as that which is disclosed under Notes * and t, p. 255 and 257 , are both produced by the same exciting cause, or at least by causes of the very same nature. Before inquiring, therefore, into the intimate causes or seat of these analogous affections, it may be of use to compare the indistinct images in both with one another, and with their respective effects, in order to detect, if possible, any physical differences between them and their distinct images that may serve to account for the phænomena they produce.

When rays of light from a primary coloured object are intercepted by the retina before they reach their focal point, the image is rendered indistinct by the diffusion and mixing of rays from single points of the object over many points of the retina. Rays from a white object similarly intercepted, have, in addition to this cause of indistinctness, another, arising from

Third Series. Vol. 1. No. 5. Nov. 1832. 


\section{$346 \mathrm{Mr}$. T. Smith's Investigation of certain Phænomena of}

the chromatic aberration; for every white image falling on the retina before or behind its true focal point is surrounded by a red or violet border, which, as well as the other cause, interferes with its distinctness. Now it is certainly a very extraordinary circumstance, that diminished sensibility to red light around the white image should occur then, and then only, when it is surrounded by this red or violet border. A distinct red border around a distinct bright object produces no such effects, as I have proved by experiments carefully made: it follows, therefore, incontrovertibly, that it is not the physical difference between a distinct and an indistinct white image that excites those changes in the sensibility which have been proved to occur. In regard to a primary coloured image, the difference between it when distinct and when indistinct, consists in that diffusion and mixing of the rays in the latter which has been noticed above, and which not only obscure the outline, but the whole surface. If these scattered rays, therefore, produce the changes in the sensibility that take place in these circumstances, we must be compelled to acknowledge that the same physical cause produces directly contrary effects at the same time; for, if this be true, the scattered rays that obscure the surface of the primary coloured image, increase the sensibility, in a remarkable degree, to the same kind of light, and the scattered rays that obscure the outline, diminish, in the same degree, the sensibility to the same kind of light. The supposition is manifestly absurd, and therefore we return with increased confidence to our first conclusions, that indistinctness of a rohite image is the true exciting cause of the diminished sensibility to red light that takes place around it in the exposed eye, and of the increased sensibility to red light that occurs in the other eye at the same time; and that indistinctness of a primary coloured image is the real exciting cause of the increased sensibility to that colour which ensues to the image itself, and the diminished sensibility to the same colour that occurs for some considerable space around the image.

The nature of the effects and the true exciting causes of these remarkable affections of sight being ascertained, it only remains to investigate the seat and nature of the actions excited by the indistinctness of the image on the retina.

In the first place, then, the retina, though it has been customary to consider it as the seat of any changes in the sensibility to light, cannot, in these cases, be regarded as the seat of either of these affections in the exposed eye ; for it is inconceivable that undulatory motions, extending from the part of the retina on which the bright light falls, to all parts around it, can be produced by an indistinct image, when a distinct 
image of equal or superior brilliancy produces no such effect: besides, it has been shown above, that to ascribe the state of the sensibility in and around the part of the retina where the bright light falls, to the physical impulse of the difference between a distinct and an indistinct image, would be to assert a physical absurdity; it follows unavoidably, therefore, that the changes in the sensibility in the exposed eye arise from actions $a b$ interno. With regard to the state of the sensibility in the unexposed eye in one of these affections, there cannot be two opinions: it must be produced by an action from within; and though we are not yet prepared to say in what manner, or by what medium, an action of the brain can affect the sensibility to light, yet the fact is a most important one; and the perfectly correct manner in which the defect of sensibility in the exposed eye is balanced by an excess of sensibility in the unexposed eye, not only affords an additional argument for their common origin, but seems to open a path, which, if duly followed, may lead to interesting discoveries in this obscure department of physiology. In the other affection of sight, though the changes in the sensibility are confined to the exposed eye only, yet the same correct balance of excess and defect is found to exist, - a circumstance that strongly corroborates the conclusion, that both of these remarkable affections of vision are produced by one common principle, and arise from one common seat. But, in the second place, the exciting causes of these affections are of such a nature, I should humbly submit, as to render them totally incapable of producing any but functional actions preorganized for the occasion. Indistinctness of image (disregarding its physical causes, which have been shown to be inadequate to the effects, ) is a purely negative quality, and can have no effects beyond the simple perception of it, except in so far as distinct vision is the end to which the mechanism of its several organs, viz. the eye, optic nerve, and brain, has been adapted: that of these three the brain is the directing organ in another highly interesting function of vision, which is also excited into action by indistinctness of image, admits of another kind of proof: the function I mean is that by which the eyes are accommodated to the different distances of objects. Suppose, after looking at a distant object, that we wish to view one near at hand, what we do in this case is to direct the two eyes so as to make their axes meet in the object to be viewed. If the former accommodation of the eyes continued, the object now viewed would be indistinct; but to prevent this, an action of the brain produces a change in the eye not yet sufficiently understood, by which a correct image of the object is formed on the retina. That the unknown change here mentioned is pro- 
duced by a function of the brain will not, I believe, be disputed, since injury of the brain by mechanical compression, \&c. destroys the function, and the contractions and dilatations of the iris that are observed to accompany its exercise. In this case, therefore, distinct vision is the end for which a certain function of the brain, as well as a certain mechanism of the eye, is provided; and the following considerations, suggested by the effects on the objects produced by the affections we are investigating, lead to similar conclusions in regard to the end or purpose of them. It is well known that the vision cannot be adapted in the common way to more than one distance at once. Now when the eyes are adapted in this way to objects at one distance, objects that are nearer or further off than that distance, are actually made more distinct than they would otherwise be by the operation of the two affections we have been examining. By one of these the indistinctness of a brighter object is lessened by the sensibility being increased to the colour of the object itself, and diminished to the same colour in less luminous objects around it, thus making the principal object brighter and better defined by a double contrast. By the other, the indistinctness arising from the chromatic aberration is removed by insensibility to the red or violet rays bordering the image; and as that insensibility extends over a wider space than the red or violet border occupies, the false vision thus occasioned is corrected by the sensibility to red light in the other eye being increased, in exactly the same degree as it is diminished in the exposed eye. Both affections, therefore, have the characters of perfect functions admirably contrived, it must be acknowledged, and as well adapted to produce distinct vision as can well be imagined in the circumstances.

Having thus given, in as compressed a form as I could adopt in justice to the subject, a full account of this investigation, I forbear, for the present, from making any observations on the singular nature of the cerebral functions thus detected, or on the perhaps still more singular nature of their exciting causes, thinking it due to truth, in a case that appears to involve principles entirely new, to wait the observations of competent inquirers, with whom it remains to confirm or refute, by an impartial scrutiny, the results which I have obtained. I shall, therefore, conclude with a short summary of those results.

1 st. Besides the well-known function by which the eyes are adapted, by turns, to different distances, two other functions, hitherto unknown, are occasionally called to the aid of vision.

2nd. Both of these newly observed functions are excited by 
indistinctness of vision; the one, when the indistinctness arises from undue scattering of the rays of light,-the other, when it is owing to the chromatic aberration of white light.

3rd. The organ excited in both cases is the brain; but whether, being thus excited, it does not also excite some other auxiliary organ, as in the case of the adaptation to different distances, does not yet appear.

4th. The actions excited are directed to the effect of removing, more or less, the exciting cause, and producing distinct vision.

Fochabers, 20th June, 1832.

Note.-An investigation of the remarkable phænomena described in the preceding ingenious paper, but leading to results different from those obtained by the author, will be published in the next Number of this Journal.-D. B.

LXV. On the Lower or Ganister Coal Series of Yorkshire. By John Phillips, F.G.S., Sec. Y.P.S., Assist. Sec. Brit. Association, \& c.*

THE lowest portion of the Yorkshire coal strata resting upon the millstone grit, produces comparatively but a small quantity of coal, and this, in general, not of a good quality. But no part of the coal-field is more curious in its geological relations, or more worthy of close study by those who desire to penetrate into the history of the production of coal. We may define this lowest coal series very simply, by saying that it is included between the millstone grit of Bramley beneath, and the flagstone of Elland above, having a thickness of about 120 or 150 yards, and inclosing near the bottom two thin seams of coal, one or both of them. workable, and several other layers scattered through its mass, too thin to be worth working. The most regular and continuous of all these coal seams reaches, in a few places, the thickness of 27 or 30 inches, but is generally only about 16 inches. It is worked at Yeadon, Rawdon, and Horsforth, near Leeds; ; at Baildon, and Heaton, near Bradford; Catharine Sluck, and Swan Banks, near Halifax; Bullhouses, near Penistone; and at several points about Sheffield.

It would have been impossible to have traced so thin a seam of coal along so extensive a range without some peculiar facilities, - some points of reference more distinct than the varying quality of the coal, and the still more irregular fluctuations of the sandstones and shales. This coal seam is covered by a roof unlike that of any other coal bed, above the mountain

* Read before the Yorkshire Philosophical Society, October 2, 1832; and communicated by the Council of that Society. 